\title{
Reconfigurable two-stage Nyquist filters utilizing the Farrow structure
}

Amir Eghbali and Håkan Johansson

\section{Linköping University Post Print}

N.B.: When citing this work, cite the original article.

(C2012 IEEE. Personal use of this material is permitted. However, permission to reprint/republish this material for advertising or promotional purposes or for creating new collective works for resale or redistribution to servers or lists, or to reuse any copyrighted component of this work in other works must be obtained from the IEEE.

Amir Eghbali and Håkan Johansson, Reconfigurable two-stage Nyquist filters utilizing the Farrow structure, 2012, IEEE Int. Symp. Circuits Syst..

http://dx.doi.org/10.1109/ISCAS.2012.6272000

Postprint available at: Linköping University Electronic Press

http://urn.kb.se/resolve?urn=urn:nbn:se:liu:diva-84864 


\title{
Reconfigurable Two-Stage Nyquist Filters Utilizing the Farrow Structure
}

\author{
Amir Eghbali and Håkan Johansson \\ Division of Electronics Systems, Department of Electrical Engineering \\ Linköping University, Sweden. E-mail: \{amire,hakanj\}@isy.liu.se.
}

\begin{abstract}
This paper introduces reconfigurable two-stage finite-length impulse response (FIR) Nyquist filters. In both stages, the Farrow structure realizes reconfigurable lowpass linear-phase FIR Nyquist filters. By adjusting the variable multipliers of the Farrow structure, various FIR Nyquist filters and integer interpolation/decimation structures are obtained, online. However, the filter design problem is solved only once, offline. Design examples illustrate the method.

Index Terms-Nyquist Filter, Farrow Structure.
\end{abstract}

\section{INTRODUCTION}

Nyquist ( $L$ th-band) filters find applications in, e.g., filter banks, spectrum sensing, pulse shaping, and timing/carrier recovery [1]-[3]. Interpolation/decimation is composed of anti-imaging/anti-aliasing filters and upsamplers/downsamplers. With Nyquist filters, we can exactly recover the input signal [4]. Efficient design of Nyquist filters is thus a necessity in communication systems.

In addition, communication engineers aim to design reconfigurable systems for multistandard communications. This leads to supporting different bandwidths and sampling rate conversion (SRC) structures which can, in principle, be handled using dedicated blocks for each standard. However, this would then require to either (i) design a large set of filters offline, or (ii) design the filters online. This is not desirable because of the resulting high complexity in both design and realization. We hence need structures which dynamically perform $\mathrm{SRC}$ at a low cost. This necessitates reconfigurable filters which also require a low arithmetic complexity.

With a noncausal Nyquist filter $H(z)$ of order $N$, we have

$$
T(z)=\sum_{l=0}^{L-1} H\left(z W_{L}^{l}\right)=1, \quad W_{L}=e^{-j \frac{2 \pi}{L}} .
$$

In the time domain,

$$
h(n)= \begin{cases}\frac{1}{L} & n=0 \\ 0 & n=m L \\ \text { arbitrary } & n \neq m L .\end{cases}
$$

A classical solution to $h(n)$ is the root-raised cosine pulse [5] but solutions based on optimization are generally more efficient [2], [6].

The SRC can be performed in single or multiple stages [4]. In multi-stage realizations, the overall SRC ratio is factorized into multiple ratios thereby reducing the arithmetic complexity [7]. However, the constraints on the overall anti-imaging/anti-aliasing filters do not change and the analysis method can hence be extended from the single-stage to the multi-stage case.

This paper proposes reconfigurable structures for two-stage realization [7] of finite-length impulse response (FIR) Nyquist filters. The Farrow structure is used in both stages so as to obtain reconfigurable Nyquist filters [8]. The zeroth polyphase component of the filters, in each stage, is a pure delay. The remaining polyphase components are realized by the Farrow structure. Therefore, both stages can be reconfigured, online. By combining these reconfigurable stages, we

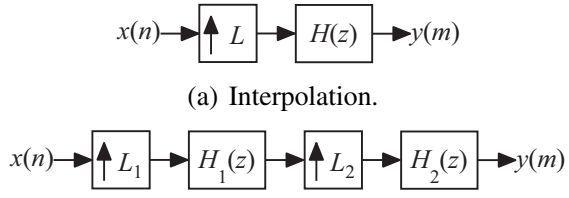

(b) Decimation

Fig. 1. Interpolation by $L$ using single-stage and two-stage structures.

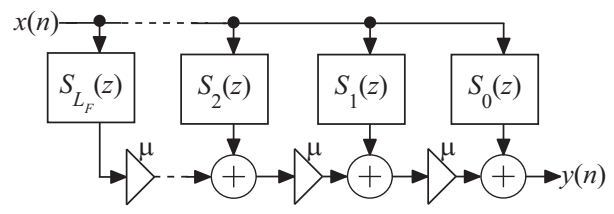

Fig. 2. Farrow structure with fixed subfilters $S_{k}(z)$ and variable FD $\mu$.

can perform reconfigurable two-stage SRC with a low arithmetic complexity. This reconfigurability does not need filter redesign. This paper only discusses the structures for interpolation because a decimator can be obtained by transposing the corresponding interpolator.

Section II discusses the single-stage SRC, two-stage SRC, and the Farrow structure. The reconfigurable two-stage Nyquist filters are outlined in Section III. In Section IV, the filter design is treated and some design examples are provided. Some discussion about the arithmetic complexity is given in Section V with the concluding remarks outlined in Section VI.

\section{PRerequisites}

As seen in Fig. 1(a), interpolation by $L$ requires an upsampler and a lowpass anti-imaging filter $H(z)$ so that $Y(z)=X\left(z^{L}\right) H(z)$. The filter $H(z)$ has a lowpass characteristic with a roll-off of $0 \leq \rho \leq 1$. The passband and stopband edges are

$$
\omega_{c} T=\pi \frac{1-\rho}{L}, \quad \omega_{s} T=\pi \frac{1+\rho}{L} .
$$

Figure 1(a) is the single-stage equivalent of Fig. 1(b) where $L=$ $L_{1} L_{2}$ [4]. Using the noble identities, the equivalent lowpass filter is

$$
H(z)=H_{1}\left(z^{L_{2}}\right) H_{2}(z) \text {. }
$$

Like (1), if $H(z)$ is a noncausal Nyquist filter, we have

$$
T(z)=\sum_{l=0}^{L-1} H_{1}\left(z^{L_{2}} W_{L}^{l L_{2}}\right) H_{2}\left(z W_{L}^{l}\right)=1 .
$$

\section{A. Farrow Structure}

The Farrow structure, shown in Fig. 2, is composed of fixed linearphase FIR subfilters $S_{k}(z), k=0,1, \ldots, L_{F}$, and it can approximate reconfigurable fractional delay (FD) filters. If $\mu$ is the FD value, the transfer function is [9] 


$$
F(z, \mu)=\sum_{k=0}^{L_{F}} S_{k}(z) \mu^{k}, \quad|\mu| \leq 0.5 .
$$

The $S_{k}(z)$ are designed so that $F(z, \mu)=z^{-\mu}$ [10]. For simplicity, the rest of the paper uses $F(z)$ instead of $F(z, \mu)$.

\section{ReCONFIgurable Two-Stage SRC}

This section considers $H_{1}(z)$ and $H_{2}(z)$ to have orders $N_{1}$ and $N_{2}$, respectively. The Type I polyphase decomposition of $H_{1}(z)$ is

$$
H_{1}(z)=\sum_{m=0}^{L_{1}-1} z^{-m} H_{1, m}\left(z^{L_{1}}\right) .
$$

If $H_{1}(z)$ is an ideal causal lowpass filter of order $N_{1}$, we have

$$
H_{1}(z)= \begin{cases}z^{-\frac{N_{1}}{2}} & \text { in the passband } \\ 0 & \text { in the stopband. }\end{cases}
$$

From (7) and (8), we get

$$
H_{1, m}(z)= \begin{cases}z^{-\frac{N_{1}}{2}-m} \frac{\text { in the passband }}{L_{1}} & \text { in the stopband. } \\ 0 & \text { in }\end{cases}
$$

A general $N_{1}$-th order causal Nyquist filter can thus be designed if

$$
H_{1,0}(z)=z^{-\frac{N_{1,0}}{2}}
$$

and by utilizing the Farrow structure to realize $H_{1, m}(z), m=$ $1,2, \ldots, L_{1}-1$, of $\operatorname{odd}^{1}$ order $N_{F_{1}}$ as [8], [11], [12]

$$
N_{1,0}=\frac{N_{1}}{L_{1}}=N_{F_{1}}+1 .
$$

Then, we can use (6) to obtain

$$
H_{1, m}(z)=\sum_{k=0}^{L_{F_{1}}} S_{1, k}(z) \mu_{1, m}^{k}
$$

with

$$
\mu_{1, m}=\frac{-m}{L_{1}}+\frac{1}{2} \Rightarrow \mu_{1, m}=-\mu_{1, L_{1}-m} .
$$

From (7), (10), and (12), we have

$$
H_{1}(z)=z^{-\frac{L_{1} N_{1,0}}{2}}+\sum_{m=1}^{L_{1}-1} z^{-m} \sum_{k=0}^{L_{F_{1}}} S_{1, k}\left(z^{L_{1}}\right) \mu_{1, m}^{k} .
$$

The same principle, as in (7)-(14), can be applied so that

$$
H_{2}(z)=z^{-\frac{L_{2} N_{2,0}}{2}}+\sum_{m=1}^{L_{2}-1} z^{-m} \sum_{k=0}^{L_{F_{2}}} S_{2, k}\left(z^{L_{2}}\right) \mu_{2, m}^{k}
$$

where

$$
N_{2,0}=\frac{N_{2}}{L_{2}}=N_{F_{2}}+1
$$

and

$$
\mu_{2, m}=\frac{-m}{L_{2}}+\frac{1}{2} \Rightarrow \mu_{2, m}=-\mu_{2, L_{2}-m} .
$$

With (4), (5), (14), and (15), some manipulations give (18) and (19) on the next page. The filter $H(z)$, in (4), is a Type I linear-phase FIR filter of order

$$
N=L_{2} N_{1}+N_{2}=L_{2} L_{1}\left(N_{F_{1}}+1\right)+L_{2}\left(N_{F_{2}}+1\right) .
$$

Here, each filter $H_{u}(z), u=1,2$, is a Nyquist ( $L_{u}$ th-band) filter, as in [7], leading to

$$
H_{u, 0}(z)=z^{-\frac{N_{u, 0}}{2}} .
$$

${ }^{1}$ With proper modifications, even-order filters can also be designed [8].

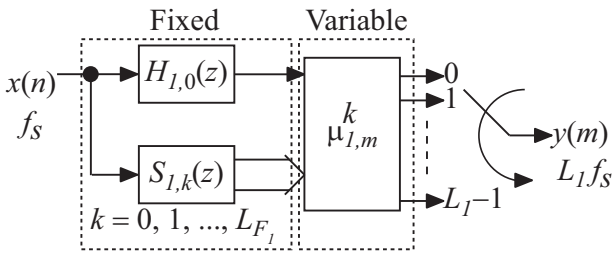

Fig. 3. Efficient interpolation by variable integer ratio $L_{1}$ using fixed subfilters, variable multipliers, and commutators.

Considering (13) and (17), we have [8], [11], [12]

$H_{1, m}(z)=\Phi_{1, m}(z)+\Psi_{1, m}(z), H_{1, L_{1}-m}(z)=\Phi_{1, m}(z)-\Psi_{1, m}(z)$,

where

$$
\begin{aligned}
\Phi_{1, m}(z) & =\sum_{k=0}^{\left\lfloor\frac{L_{F_{1}}}{2}\right\rfloor} S_{1,2 k}(z) \mu_{1, m}^{2 k}, \\
\Psi_{1, m}(z) & =\sum_{k=1}^{\left\lfloor\frac{L_{F_{1}}+1}{2}\right\rfloor} S_{1,2 k-1}(z) \mu_{1, m}^{2 k-1} .
\end{aligned}
$$

As in Fig. 3, reconfigurable SRC by $L_{1}$ requires fixed filters $S_{1, k}(z)$ and $H_{1,0}(z)$, variable multipliers $\mu_{1, m}^{k}$, and commutators [8], [11], [12]. Consequently, reconfigurable two-stage interpolation by $L=$ $L_{1} L_{2}$ can be realized according to Fig. 4 .

\section{FILTER DESIGN}

With noncausal ideal filters, we have

$$
H(z)= \begin{cases}1 & \text { in the passband } \\ 0 & \text { in the stopband. }\end{cases}
$$

With nonideal filters, we can approximate (25). This section treats the minimax design problem as

$$
\min \quad \delta \text { subject to }\left|H\left(e^{j \omega T}\right)\right| \leq \delta, \quad \omega T \in \Omega
$$

and we consider two cases where

Case 1: $\Omega=\left[\omega_{s} T, \pi\right]$

Case 2: $\quad \Omega=\bigcup_{p=1}^{\lfloor L / 2\rfloor}\left[\frac{(2 p-1+\rho) \pi}{L}, \min \left(\frac{(2 p+1-\rho) \pi}{L}, \pi\right)\right]$.

Here, Case 1 covers the whole stopband from $\omega_{s} T$ up to $\pi$ but Case 2 excludes the don't-care bands centered on $\frac{(2 p+1) \pi}{L}, p=$ $1,2, \ldots,\left\lfloor\frac{L-1}{2}\right\rfloor$ for $L>2$. This is admissible in some applications [7]. Note that in a Nyquist filter, the passband and stopband ripples are related to each other [7], [13].

In (26), the free optimization parameters are the coefficients of $S_{u, k}(z)$ and $H_{u, 0}(z)$ with $u=1,2$, and $k=1,2, \ldots, L_{F_{u}}$. During the filter design, the values of $\mu_{u, m}^{k}, L_{u}, N_{F_{u}}$, and $L_{F_{u}}$ are pre-determined. After solving (26) only once, we can realize reconfigurable FIR Nyquist filters. For this realization, the impulse responses of $S_{u, k}(z)$ and $H_{u, 0}(z)$ as well as the values of $N_{F_{u}}$ and $L_{F_{u}}$ are fixed. However, only the values of $\mu_{u, m}^{k}$ and $L_{u}$ need to be adjusted, online.

Here, the design problem for each stage is a convex optimization problem [8] where, ideally, $S_{u, k}\left(e^{j \omega T}\right)=\frac{(-j \omega T)^{k}}{k !}$ [8]. This can be used to find the initial solutions, using the linprog routine of MATLAB. These initial solutions can then be used to solve the general nonlinear problem of (26), using the fminimax routine of MATLAB 


$$
\begin{array}{r}
H(z)=\left(z^{-\frac{L N_{1,0}}{2}}+\sum_{m=1}^{L_{1}-1} z^{-m L_{2}} \sum_{k=0}^{L_{F_{1}}} S_{1, k}\left(z^{L}\right) \mu_{1, m}^{k}\right)\left(z^{-\frac{L_{2} N_{1,0}}{2}}+\sum_{m=1}^{L_{2}-1} z^{-m} \sum_{k=0}^{L_{F_{2}}} S_{1, k}\left(z^{L_{2}}\right) \mu_{2, m}^{k}\right) . \\
T(z)=\sum_{l=0}^{L-1}\left(z^{-\frac{L N_{1,0}}{2}}+\sum_{m=1}^{L_{1}-1}\left(z W_{L}^{l}\right)^{-m L_{2}} \sum_{k=0}^{L_{F_{1}}} S_{1, k}\left(z^{L}\right) \mu_{1, m}^{k}\right)\left(\left(z W_{L}^{l}\right)^{-\frac{L_{2} N_{1,0}}{2}}+\sum_{m=1}^{L_{2}-1}\left(z W_{L}^{l}\right)^{-m} \sum_{k=0}^{L_{F_{2}}} S_{1, k}\left(\left(z W_{L}^{l}\right)^{L_{2}}\right) \mu_{2, m}^{k}\right) .
\end{array}
$$

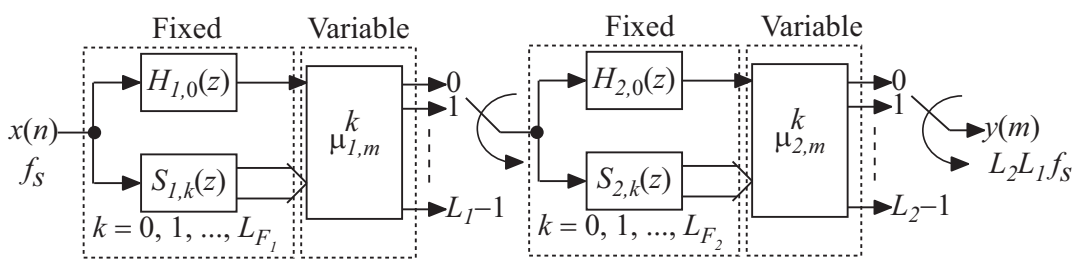

Fig. 4. Efficient interpolation by variable integer ratio $L=L_{2} L_{1}$ using fixed subfilters, variable multipliers, and commutators..
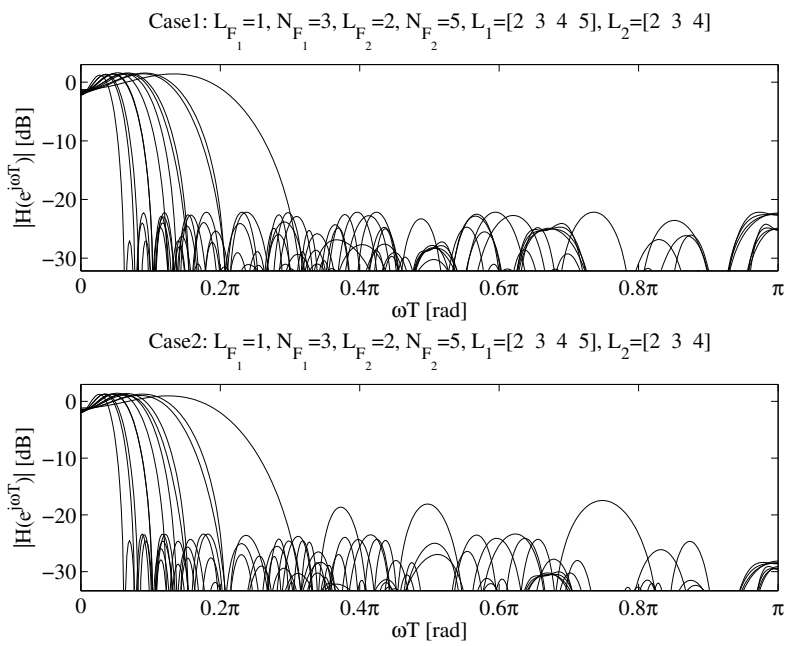

Fig. 5. Characteristics of $\left|H\left(e^{j \omega T}\right)\right|$ for reconfigurable Nyquist filters.

\section{A. Design Examples}

Figures 5-8 show the characteristics of some reconfigurable Nyquist filters obtained from (26) where $\rho=0.2$. As can be seen, we can exactly meet (1). Further, we can decrease the stopband ripple by increasing $L_{F_{1}}, L_{F_{2}}, N_{F_{1}}$, and $N_{F_{2}}$. Also, allowing don't-care bands helps further decrease $\delta$, in (26), without increasing the values of $L_{F_{1}}, L_{F_{2}}, N_{F_{1}}$, and $N_{F_{2}}$. In Figs. 5(a) and 5(b), the values of $\delta$ are, respectively, $7.7981 \times 10^{-2}$ and $6.7892 \times 10^{-2}$. As can be seen from Figs. 5(b) and 7(b), the magnitude response, in don't-care bands, is exceeding the corresponding $\delta$. For Figs. 7(a) and 7(b), the values of $\delta$ are, respectively, $4.7369 \times 10^{-3}$ and $3.8423 \times 10^{-3}$.

\section{ARithmetic Complexity}

For interpolation by $L_{u}, u=1,2$, using a Nyquist filter $H_{u}(z)$ which is realized with a Farrow structure having $L_{F_{u}}$ subfilters of orders $N_{F_{u}}$, we need [8]

$$
C_{u}= \begin{cases}\frac{\left(L_{F_{u}}+1\right)\left(N_{F_{u}}+1\right)}{2}+\frac{L_{F_{u}}\left(L_{u}-1\right)}{2} & \text { odd } L_{u} \\ \frac{\left(L_{F_{u}}+1\right)\left(N_{F_{u}}+1\right)}{2}+\frac{L_{F_{u}}\left(L_{u}-2\right)}{2} & \text { even } L_{u}\end{cases}
$$

distinct multiplications per input sample. Therefore, the total number of distinct multiplications, for reconfigurable two-stage SRC by $L=$
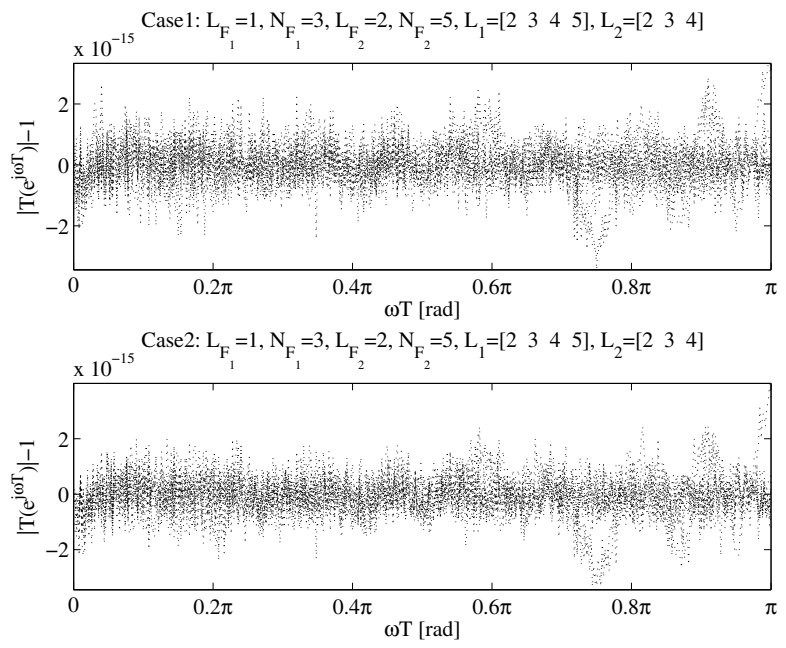

Fig. 6. Characteristics of $\left|T\left(e^{j \omega T}\right)\right|-1$ for reconfigurable Nyquist filters.

$L_{1} L_{2}$, becomes

$$
C_{\mathrm{rt}}=\left(C_{1}+L_{1} C_{2}\right) .
$$

In the conventional fixed two-stage case [7], one can utilize fixed $L_{u}$ th-band filters $H_{u}(z), u=1,2$, so as to obtain fixed Nyquist filters $H(z)$. Then, the total number of distinct multiplications becomes

$C_{\mathrm{ct}}=\left(\frac{N_{1}}{2}-\left\lfloor\frac{\frac{N_{1}}{2}-1-k_{1}}{L_{1}}\right\rfloor\right)+L_{1}\left(\frac{N_{2}}{2}-\left\lfloor\frac{\frac{N_{2}}{2}-1-k_{2}}{L_{2}}\right\rfloor\right)$ where $k_{u}=\frac{N_{u}}{2} \bmod L_{u}$ with $\frac{N_{u}}{2} \bmod L_{u}$ being the remainder of $\frac{N_{u}}{2 L_{u}}$. Also, $\lfloor$.$\rfloor represents the floor operation. In (30), the terms inside$ parentheses refer to the number of distinct nonzero coefficients in a general $L_{u}$ th-band filter of order $N_{u}$.

Tables I and II summarize the results of the designs in Fig. 7(a) using, respectively, reconfigurable and the conventional fixed twostage Nyquist filters. In Table I, we need a maximum of 141 distinct multiplications to simultaneously realize $L$ th-band filters with $L=\{4,6,8,9,10,12,15,16,20\}$. On the other hand, Table II will require $\sum C_{c t}=375$ distinct multiplications to realize all of these Nyquist filters. This shows a $62 \%$ reduction of the arithmetic 

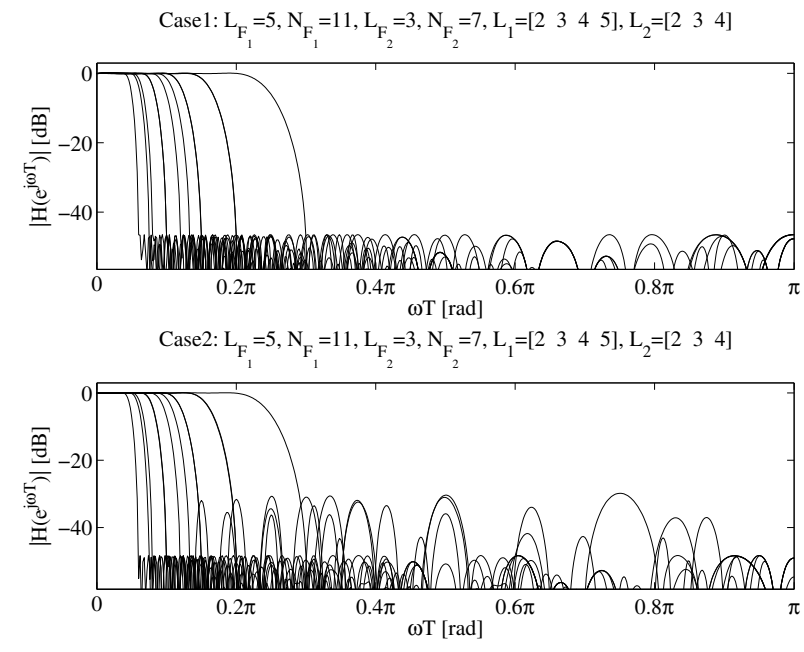

Fig. 7. Characteristics of $\left|H\left(e^{j \omega T}\right)\right|$ for reconfigurable Nyquist filters.
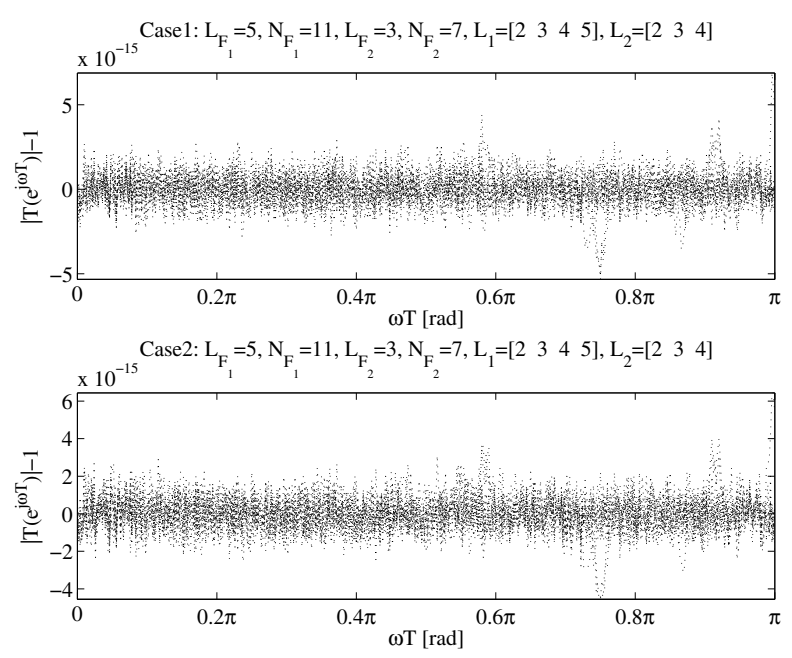

Fig. 8. Characteristics of $\left|T\left(e^{j \omega T}\right)\right|-1$ for reconfigurable Nyquist filters.

complexity while obtaining a reconfigurability. Note also that Table I only requires to save the coefficients of $S_{u, k}(z)$ and $H_{u, 0}(z)$ but Table II needs to save all of the coefficients for $H_{1}(z)$ and $H_{2}(z)$, i.e., $\sum N_{1}+N_{2}$. This also means that the reconfigurable two-stage design has fewer optimization parameters.

\section{CONCLuSion}

Reconfigurable two-stage Nyquist filters, using the Farrow structure, were outlined. These Nyquist filters are obtained by one offline filter design and through adjusting (i) the number of polyphase components, and (ii) the variable multipliers of the Farrow structure. In comparison to the conventional fixed two-stage Nyquist filters, the arithmetic complexity (in terms of the number of distinct multiplications) is reduced by $62 \%$.

\section{REFERENCES}

[1] B. P. Lathi, Modern Digital and Analog Communication Systems. Oxford University Press, 2009.

[2] K. Väisänen and M. Renfors, "Efficient digital filters for pulse-shaping and jitter-free frequency error detection and timing recovery," Signal Processing, vol. 81, no. 4, pp. 829-844, Apr. 2001.
TABLE I

PARAMETERS FOR RECONFIGURABLE TWO-STAGE DESIGNS IN Fig. 7(A)

\begin{tabular}{|c|c|c|c|c|c|}
\hline$L$ & $L_{1}$ & $L_{2}$ & $N_{1}$ & $N_{2}$ & $C_{r t}$ \\
\hline 4 & 2 & 2 & 24 & 16 & 68 \\
\hline 6 & 2 & 3 & 24 & 24 & 74 \\
\hline 8 & 2 & 4 & 24 & 32 & 74 \\
\hline 6 & 3 & 2 & 36 & 16 & 89 \\
\hline 9 & 3 & 3 & 36 & 24 & 98 \\
\hline 12 & 3 & 4 & 36 & 32 & 98 \\
\hline 8 & 4 & 2 & 48 & 16 & 105 \\
\hline 12 & 4 & 3 & 48 & 24 & 117 \\
\hline 16 & 4 & 4 & 48 & 32 & 117 \\
\hline 10 & 5 & 2 & 60 & 16 & 126 \\
\hline 15 & 5 & 3 & 60 & 24 & 141 \\
\hline 20 & 5 & 4 & 60 & 32 & 141 \\
\hline
\end{tabular}

TABLE II

PARAMETERS FOR CONVENTIONAL FIXED TWO-STAGE TO MEET THE SPECIFICATIONS OF FIG. 7(A)

\begin{tabular}{|c|c|c|c|c|c|}
\hline$L$ & $L_{1}$ & $L_{2}$ & $N_{1}$ & $N_{2}$ & $C_{c t}$ \\
\hline 4 & 2 & 2 & 26 & 10 & 16 \\
\hline 6 & 2 & 3 & 22 & 14 & 19 \\
\hline 8 & 2 & 4 & 22 & 14 & 21 \\
\hline 6 & 3 & 2 & 34 & 6 & 22 \\
\hline 9 & 3 & 3 & 34 & 10 & 28 \\
\hline 12 & 3 & 4 & 34 & 12 & 31 \\
\hline 8 & 4 & 2 & 46 & 6 & 31 \\
\hline 12 & 4 & 3 & 46 & 8 & 35 \\
\hline 16 & 4 & 4 & 46 & 12 & 43 \\
\hline 10 & 5 & 2 & 58 & 6 & 40 \\
\hline 15 & 5 & 3 & 58 & 8 & 45 \\
\hline 20 & 5 & 4 & 56 & 6 & 44 \\
\hline
\end{tabular}

[3] B. Farhang-Boroujeny, "Filter bank spectrum sensing for cognitive radios," IEEE Trans. Signal Processing, vol. 56, no. 5, pp. 1801-1811, May 2008.

[4] P. P. Vaidyanathan, Multirate Systems and Filter Banks. Englewood Cliffs, NJ: Prentice-Hall, 1993.

[5] S. D. Assimonis, M. Matthaiou, and G. K. Karagiannidis, "Twoparameter Nyquist pulses with better performance," IEEE Commun. Lett., vol. 12, no. 11, pp. 807-809, Nov. 2008.

[6] B. Farhang-Boroujeny and G. Mathew, "Nyquist filters with robust performance against timing jitter," IEEE Trans. Signal Processing, vol. 46, no. 12, pp. 3427-3431, Dec. 1998.

[7] T. Saramäki and Y. Neuvo, "A class of FIR Nyquist ( $N$ th-band) filters with zero intersymbol interference," IEEE Trans. Circuits Syst., vol. 34, no. 10, pp. 1182-1190, Oct. 1987.

[8] H. Johansson and O. Gustafsson, "Linear-phase FIR interpolation, decimation, and $M$-th band filters utilizing the Farrow structure," IEEE Trans. Circuits Syst. I, vol. 52, no. 10, pp. 2197-2207, Oct. 2005.

[9] C. W. Farrow, "A continuously variable digital delay element," in Proc. IEEE Int. Symp. Circuits Syst., vol. 3, Espoo, Finland, June 1988, pp. 2641-2645.

[10] H. Johansson and P. Löwenborg, "On the design of adjustable fractional delay FIR filters," IEEE Trans. Circuits Syst. II, vol. 50, no. 4, pp. 164169, Apr. 2003

[11] A. Eghbali, H. Johansson, and P. Löwenborg, "A Farrow-structure-based multi-mode transmultiplexer," in Proc. IEEE Int. Symp. Circuits Syst., Seattle, Washington, USA, May 2008, pp. 3114-3117.

[12] — "A class of multimode transmultiplexers based on the Farrow structure," Circuits Syst. Signal Processing, 2011, accepted.

[13] A. Eghbali, T. Saramäki, and H. Johansson, "On two-stage Nyquist pulse shaping filters," IEEE Trans. Signal Processing, vol. 60, no. 1, pp. 483488, Jan. 2012. 